\title{
Densificación y estratificación social en Bogotá: distribución sesgada de la inversión privada
}

Juan G. Yunda. Pontificia Universidad Javeriana, Bogotá, Colombia.

RESUMEN | Las ciudades latinoamericanas se caracterizan por su segregación social y desajuste espacial. No obstante, pocos estudios analizan cómo se reproducen estos fenómenos a través de la política pública. En Colombia se implementó un sistema denominado "estratificación socioeconómica", basado en las características físicas de las viviendas y su entorno inmediato, cuyo objetivo fue facilitar la ejecución de subsidios cruzados para el pago de servicios públicos. Sin embargo, ha sido asimilado por parte de la población como indicador de ingreso y reconocimiento social. Si bien existen estudios socioeconómicos que indican las deficiencias del sistema de estratificación señalado, no hay muchos que exploren su impacto en el desarrollo urbano. En el caso de Bogotá, el análisis que aquí se presenta, realizado con métodos mixtos y que incluye datos de licencias de construcción y entrevistas, encontró que los patrones geográficos de densificación de la ciudad estuvieron determinados por la estratificación más que por los objetivos planteados en el Plan de Ordenamiento Territorial.

PALABRAS CLAVE | concentración espacial, segregación, urbanismo.

ABSTRACT | Latin American cities are known for their patterns of social segregation and spatial mismatch. However, few studies have analyzed how public policy may reproduce these patterns. Authorities implemented in Colombia a system of so-called Socio-Economic Stratification, based on the physical characteristics of the houses and their urban environment. This system aimed to facilitate the distribution of cross subsidies for the payment of utility bills. However, the public has assimilated stratification as an indicator of income and social recognition. Although there are sociological and economic studies that indicate how stratification has deficiencies, few studies explore its impact on urban development. By using mixed methods and including data about building permits and interviews, for the case of Bogotá, this analysis found that the stratification indicator determined the geographic patterns of densification of the city, rather than the objectives portrayed in the Comprehensive Plan.

KEYWORDS | spatial concentration, segregation, urbanism. 


\section{Introducción}

Las ciudades latinoamericanas se caracterizan por una profunda segregación socioespacial. Esta conformación se hace evidente en las diferencias en la morfología urbana, en las tipologías de vivienda y en la distribución geográfica de los nodos comerciales y de empleo. Mientras los grupos de alto ingreso habitan en lugares urbanizados formalmente, donde cuentan con suficientes calles, parques, plazoletas y servicios públicos; los sectores de bajo ingreso lo hacen en lugares urbanizados informalmente, sin redes de circulación ni espacio público suficiente, además de estar ubicados frecuentemente en áreas bajo amenaza de inundaciones o deslizamientos de tierra. En las zonas de alto ingreso, las viviendas fueron construidas con estándares arquitectónicos y urbanísticos que permiten una suficiente iluminación, ventilación y oferta de estacionamientos. Contrariamente, los grupos de bajo ingreso habitan en viviendas autoconstruidas, o desarrolladas con muy bajos estándares de construcción, frecuentemente oscuras y poco ventiladas en sus espacios internos, además de vulnerables ante incendios y movimientos sísmicos. Finalmente, las zonas de alto ingreso en las ciudades latinoamericanas están frecuentemente localizadas en áreas cercanas o bien conectadas a los centros de empleo, educativos y de servicios de mayor calidad, mientras los grupos de bajo ingreso tienen que localizarse en áreas periféricas donde tienen mayores costos en dinero y tiempo para acceder a las oportunidades económicas. La persistencia de estos patrones de segregación socioespacial es importante debido a que la excesiva localización centralizada de la población de alto ingreso $-\mathrm{y}$, por ende, de las actividades económicas en el sector terciario- ha encarecido la vivienda conectada a los distritos de negocios e impedido el acceso a nuevas oportunidades económicas para la población de más bajo ingreso (Guzmán, Oviedo \& Bocarejo, 2017; Suárez \& Delgado, 2009; Suárez-Lastra \& Delgado-Campos, 2007). Se trata de un fenómeno llamado en la literatura inglesa spatial mismatch, o "desajuste espacial" (Kain, 1968).

Estas diferencias han sido ya muy discutidas en la literatura. Sin embargo, pocos estudios han explorado cómo los discursos de planeación y las normas urbanísticas contribuyen a perpetuarlas. En este campo, son pioneros para el caso colombiano los trabajos de Blanco (2012) y Koch (2015). Por un lado, Blanco propone, analizando la historia de la normativa urbana de Bogotá, que las diferencias socioespaciales son resultado de la reducción de todos los discursos de planificación a uno solo, que él llama land entrepreneurialism o "emprendimiento basado en la tierra". De acuerdo con Blanco, este discurso busca sacar el mayor provecho privado de la propiedad de la tierra, por encima de cualquier criterio técnico o de equidad social promovido por el sector público. De esta manera, un propietario de tierra busca construir en su predio, legal o ilegalmente, la mayor cantidad de espacio posible, tanto horizontal como verticalmente, para generar también la mayor cantidad de ingreso posible. Esta construcción intensiva ejerce una gran presión sobre el espacio y servicios públicos: mayor cantidad de habitantes presiona por calles, parques y plazas más grandes, e igualmente por una mayor capacidad del acueducto, alcantarillado, redes eléctricas y recolección de basura. Los costos de ampliación de esta infraestructura -o de su construcción, cuando no existe- son en mayor medida asumidos por el 
sector público. En la práctica, entonces, se promueve la privatización de los beneficios de los mercados de la tierra, con una socialización de sus costos. Blanco explica también cómo frecuentemente las normas urbanísticas privilegian ese sistema. Es lo que ocurre en Bogotá a través del sistema de estratificación socioeconómica -que será explicado con mayor profundidad más adelante-, el cual, a pesar de no ser una política de planeación, dicta normas diferenciadas de urbanismo generando un mercado segmentado de vivienda que limita la oferta y la competencia. Eventualmente, como indica Blanco (2012), estas prácticas desarrollan un mercado de alto costo y baja calidad, cautivo por monopolios formales e informales de actores dentro del sector privado (p. 30).

Mientras Blanco explica el discurso de planeación tras los procesos de segregación socioespacial, Koch se enfoca en cómo el sector privado influencia la morfología a través de las normas urbanísticas. Utiliza para ello el caso de la ciudad costera de Barranquilla, en su proceso de formulación del Plan de Ordenamiento Territorial (РОт) del año 2000. Koch encuentra que una importante compañía del país, propietaria de tierra en la periferia de la ciudad, incidió de manera significativa en el proceso de formulación del plan. Esa compañía logró que las normas urbanísticas impidieran la localización de viviendas de bajo costo en terrenos de su propiedad y privilegiaran el uso de automóvil, con el fin de destinar esos predios a desarrollos inmobiliarios para "población de estrato alto", multiplicando el valor de esa tierra sin urbanizar. Ello se efectuó a través de profesionales en planeación que, en corto tiempo, pasaban de prestar servicios en la empresa inmobiliaria de la compañía, a hacerlo para la municipalidad. Esta "puerta giratoria" facilitó el desarrollo de conjuntos de vivienda cerrados de alta densidad, sin estándares de conectividad peatonal, requerimientos de generación de vivienda de interés social, o participación ciudadana. Según Koch, procesos como el señalado promueven una forma urbana cerrada y segregada que beneficia en mayor medida a los grupos de alto ingreso, y que es frecuente en las ciudades en países en vías de desarrollo. Koch llama a este tipo de urbanismo arranged urbanism, o "urbanismo arreglado".

Inspirado en el trabajo de Blanco y Koch, el presente estudio tiene como objetivo principal comprobar la influencia del sistema de estratificación socioeconómica colombiano en el desarrollo urbano segregado de la ciudad de Bogotá en los últimos años. Para esto, se contó con recientes fuentes de información tanto cuantitativa como cualitativa, que incluyen una base de datos de licencias de construcción, documentos de planeación -como diferentes planes directores y el РОт vigente de la ciudad- y entrevistas a actores del desarrollo urbano de la ciudad, tanto del sector público como del privado. De esta manera, los objetivos específicos de este trabajo son:

- Analizar en profundidad el sistema de estratificación socioeconómica vigente en Bogotá, su evolución y las críticas que se le hacen.

- Establecer si los patrones de segregación social identificados por la política de estratificación socioeconómica a finales del siglo xx siguen vigentes en la ciudad.

- Evaluar el rol del sistema de estratificación socioeconómica en las leyes urbanísticas y de ordenamiento territorial en la ciudad. 
- Determinar, a través de la base de datos de licencias de construcción, la distribución de la actividad constructora en edificaciones para usos residenciales y no residenciales a través de los diferentes estratos socioeconómicos.

- Explorar cómo la norma urbanística local facilita la influencia del sistema de estratificación socioeconómica en el desarrollo urbano de la ciudad.

\section{Orígenes y diseño de la política de estratificación socioeconómica en Colombia}

En Colombia, la política pública de estratificación socioeconómica tiene sus orígenes en la estructura urbana desigual de las ciudades. Esta disposición se caracteriza por las grandes diferencias morfológicas entre la ciudad de origen formal que se levantó siguiendo normas de urbanismo, y los asentamientos informales que se construyeron sin normas o siguiendo normas mínimas de urbanización, modalidades opuestas que se desarrollaron en paralelo a lo largo del siglo xx. Durante las décadas de 1960 y 1970, las autoridades colombianas empezaron a preocuparse por la desigual infraestructura de esos dos tipos de ciudad. Mientras los barrios de origen formal, donde habitaban las élites y una emergente clase media, tenían toda la dotación necesaria de servicios públicos domiciliarios, la ciudad informal, donde habitaba la población más vulnerable, carecía de ellos o se suplía a través de conexiones ilegales. Tal situación llevaba a que la población más pobre de la ciudad sufriera de escasez o contaminación de agua, además de carecer de redes de alcantarillado, lo que originaba mayor incidencia de enfermedades y mayores costos para acceder a fuentes de agua limpia. En otros casos el fluido eléctrico era inestable, lo que frecuentemente dañaba los electrodomésticos o causaba incendios en las viviendas. Se trataba, así, de una situación que perpetuaba las ventajas de las élites y las condiciones de pobreza de las clases bajas.

Las autoridades colombianas decidieron, entonces, crear un mecanismo que brindara equidad al sistema de servicios públicos domiciliarios, forzando a las elites a subsidiar el costo de la infraestructura en las áreas donde vivía la población más pobre. Fue así que, al final de la década de los sesenta, el gobierno colombiano introdujo el Decreto 3069 (Presidente de la República de Colombia, 1968), que obligó a las empresas de servicios públicos a establecer un sistema de tarifas diferenciales, según fuera el nivel socioeconómico de la población de cada barrio. Sin embargo, para la época, la información sobre ingreso de los hogares era escasa en el país, el catastro estaba poco desarrollado, y ni el gobierno ni las empresas de servicios públicos podían determinar quién era pobre y quién era rico. En consecuencia, durante los años setenta y ochenta, los municipios y empresas de servicios públicos desarrollaron independientemente sistemas de estratificación diferentes, que muchas veces eran arbitrarios y no coincidían entre sí. 
TABLA I | Variables metodología de estratificación, Departamento Nacional de Planeación (DNP)

\begin{tabular}{|c|c|}
\hline \multicolumn{2}{|c|}{ FACTORES GRUPO I: CARACTERÍSTICAS DE LA VIVIENDA Y EL ENTORNO } \\
\hline Variables & Opciones \\
\hline \multirow{2}{*}{$\begin{array}{l}\text { V1: Existencia de entrada principal en } \\
\text { las viviendas de lado de manzana }\end{array}$} & Sí \\
\hline & No \\
\hline \multirow{5}{*}{$\begin{array}{l}\text { V2: Vías de acceso (la calle o la vía de } \\
\text { acceso al lado de la manzana) }\end{array}$} & Sendero o camino \\
\hline & Peatonal \\
\hline & Vehicular en tierra \\
\hline & Vehicular en recebo basto o gravilla \\
\hline & Vehicular en cemento, asfalto o adoquín \\
\hline \multirow{4}{*}{$\begin{array}{l}\text { V3: Tamańo predominante del } \\
\text { frente de las viviendas del lado de la } \\
\text { manzana }\end{array}$} & Hasta 7 metros \\
\hline & Más de 7 y hasta 9 metros \\
\hline & Más de 9 y hasta 12 metros \\
\hline & Más de 12 metros \\
\hline \multirow{3}{*}{$\begin{array}{l}\text { V4: Andén (predominancia en el lado } \\
\text { de la manzana) }\end{array}$} & Sin andén \\
\hline & Con andén sin zona verde \\
\hline & Con andén con zona verde \\
\hline \multirow{4}{*}{$\begin{array}{l}\text { V5: Antejardín (predominancia en el } \\
\text { lado de la manzana) }\end{array}$} & Sin antejardín \\
\hline & Con antejardín pequeño \\
\hline & Con antejardín mediano \\
\hline & Con antejardín grande \\
\hline \multirow{6}{*}{$\begin{array}{l}\text { V6: Garajes (predominancia en el } \\
\text { lado de la manzana) }\end{array}$} & Sin garaje ni parqueadero \\
\hline & Con garaje cubierto usado para otros fines \\
\hline & Con parqueadero o zona de parqueo \\
\hline & Con garaje adicionado a la vivienda \\
\hline & Con garaje sencillo que hace parte del diseño original de la vivienda \\
\hline & Con garajes dobles o en sótano \\
\hline \multirow{5}{*}{$\begin{array}{l}\text { V7: Material de las fachadas } \\
\text { (predominancia en el lado de la } \\
\text { manzana) }\end{array}$} & En guadua, cańa, esterilla, tabla, desechos \\
\hline & $\begin{array}{l}\text { Sin cubrir: adobe, bahareque, tapia pisada, placa prefabricada, } \\
\text { bloque o ladrillo común }\end{array}$ \\
\hline & En revoque, pañete o repello, sin pintura \\
\hline & En revoque, pańete o repello con pintura \\
\hline & Con enchapes, en ladrillo pulido o en madera fina. \\
\hline \multirow{4}{*}{$\begin{array}{l}\text { V8: Material de los techos } \\
\text { (predominancia en el lado de la } \\
\text { manzana) }\end{array}$} & Desechos, telas asfálticas o pedazos de tejas \\
\hline & Placa de entrepiso \\
\hline & Terraza, azotea o cubierta sencilla \\
\hline & Lujosa u ornamental \\
\hline \multicolumn{2}{|c|}{ FACTORES GRUPO 2: CONTEXTO URBANÍSTICO } \\
\hline Variable & Opciones \\
\hline \multirow{14}{*}{$\begin{array}{l}\text { Zona por criterios de hábitat a la cual } \\
\text { pertenece la manzana }\end{array}$} & Zona de pobreza \\
\hline & Zona de tolerancia \\
\hline & Desarrollo progresivo sin consolidar \\
\hline & Deterioro urbanístico \\
\hline & Industrial \\
\hline & Desarrollo progresivo consolidado \\
\hline & Comercial predominante \\
\hline & Residencial intermedio \\
\hline & Comercial compatible \\
\hline & Residencial exclusivo \\
\hline & Residencial de baja densidad \\
\hline & Institucional \\
\hline & Lotes y otros sin vivienda \\
\hline & Zona verde \\
\hline
\end{tabular}

FUENTE SEPÚlVEdA Rico, López CAMACHo \& GALlego ACEVEdo (20I4), PP. 28-29 
Para atender esta problemática, el Departamento Nacional de Planeación (DNP) de Colombia desarrolló en 1994 una nueva metodología unificada para todo el país, a través de la Ley 142 (Congreso de Colombia, 1994). La metodología propuesta por el DNP tenía en cuenta la ausencia o mala calidad de la información catastral a nivel nacional y, por ende, establecía que la estratificación socioeconómica fuera determinada por las condiciones físicas y urbanísticas de los predios. Según establecía el DNP, todos los predios de las áreas urbanas en Colombia deberían ser clasificados en uno de seis "estratos", siendo el designado con el número 1 el más bajo, y con 6 el más alto. El estrato era determinado por una combinación de variables en dos grupos de factores, el primero relacionado con las características de la vivienda y su entorno inmediato, y el segundo con las características de su contexto urbanístico, como lo indica la tabla 1. Esta metodología condujo a la separación clara de la ciudad entre áreas desarrolladas en vivienda de expansión progresiva de autoconstrucción, y conjuntos unifamiliares o multifamiliares de alto costo, como indica la figura 1 a la izquierda.

Siguiendo el mandato de la Ley 142, la ciudad de Bogotá condujo su primer censo de Estratificación en 1997. Sin embargo, debido a su tamaño y complejidad, las autoridades locales de Bogotá alteraron la metodología. El principal cambio, que obedeció al tamańo y complejidad de la ciudad, fue la asignación del estrato por manzana, mientras que los demás municipios lo asignaron por frente de calle.

Según la Ley 142, cada municipio en Colombia debía actualizar su estratificación cada dos ańos a través de un Comité Permanente de Estratificación Socio-Económica, que aconseja al alcalde para modificar los estratos según las metodologías del DNP. Los cambios podían responder a nuevas edificaciones, a variaciones en el entorno construido o a peticiones de la ciudadanía. Entre 1999, cuando se estableció la primera estratificación, y 2016, las autoridades de Bogotá condujeron seis procesos de actualización. El último se produjo en 2013 (Alcalde Mayor de Bogotá D.C., 2013) — reproducida en el mapa a la derecha de la figura 1 - , cuando 35.000 nuevas viviendas fueron estratificadas y 37 manzanas cambiaron de estrato. De acuerdo con la Secretaría Distrital de Planeación de Bogotá D.c. (sDP, 2011b), para 2011 cerca del 50\% de la población vivía en manzanas clasificadas en los estratos 1 y 2 (asentamientos de origen informal en proceso de consolidación) y aproximadamente el 15\% de la población lo hacía en estratos altos 4, 5 y 6 (predominantemente multifamiliares o conjuntos cerrados de casas). Esta estructura coincide en una gran medida con la planteada por Griffin y Ford en su artículo de 1980 (p. 410).

\section{Críticas a la política de estratificación socioeconómica}

A pesar de que gracias a este sistema en las últimas décadas Bogotá logró indicadores de cobertura de servicios públicos cercanos al 100\% (sDp, 2015), recientemente la estratificación ha sido muy criticada en las altas esferas académicas y de gobierno, principalmente por problemas en su metodología y por su impacto social. En general, tanto las autoridades como la ciudadanía han tomado erróneamente el indicador de estratificación como un indicador de ingreso de los hogares, mientras que, como se indicó anteriormente, la metodología de la estratificación no incluye 
ningún dato de ingreso familiar. Un análisis de los datos demuestra que los indicadores de pobreza y el estrato no están necesariamente correlacionados. Por ejemplo, según datos de la SDP (2011a), en el estrato 1, el más bajo, no toda la población está en estado de pobreza, solo el 50\%, mientras que en el estrato 6, el más alto, hay un $6 \%$ de pobreza, porcentaje incluso mayor al del estrato 5 (4\%).

La SDP publicó su primer estudio en 2004, y en él cuestionaba las suposiciones de la metodología del DNP, utilizando para ello el caso de Bogotá (Departamento Administrativo de Planeación Distrital [DAPD], 2004). En esa ocasión, los autores utilizaron modelos estadísticos donde compararon datos socioeconómicos de diferentes encuestas con los indicadores de estrato. Encontraron que la determinación de seis estratos no se ajusta a la realidad del ingreso de los hogares bogotanos. Determinaron que la variación de ingreso en el estrato más alto (6) y en el más bajo (1) es muy alta, por lo cual se recomendó la creación de un nuevo estrato 7 . Más tarde, otros estudios (Aliaga-Linares \& Álvarez-Rivadulla, 2010; Ibatá-Ceballos \& Torres-Arias, 2006) compararon datos del censo nacional de 2005 con el indicador de estrato en Bogotá, encontrando una gran heterogeneidad de niveles de ingreso dentro de cada estrato.

FIGURA I | Tipología de vivienda y localización de cada Estrato
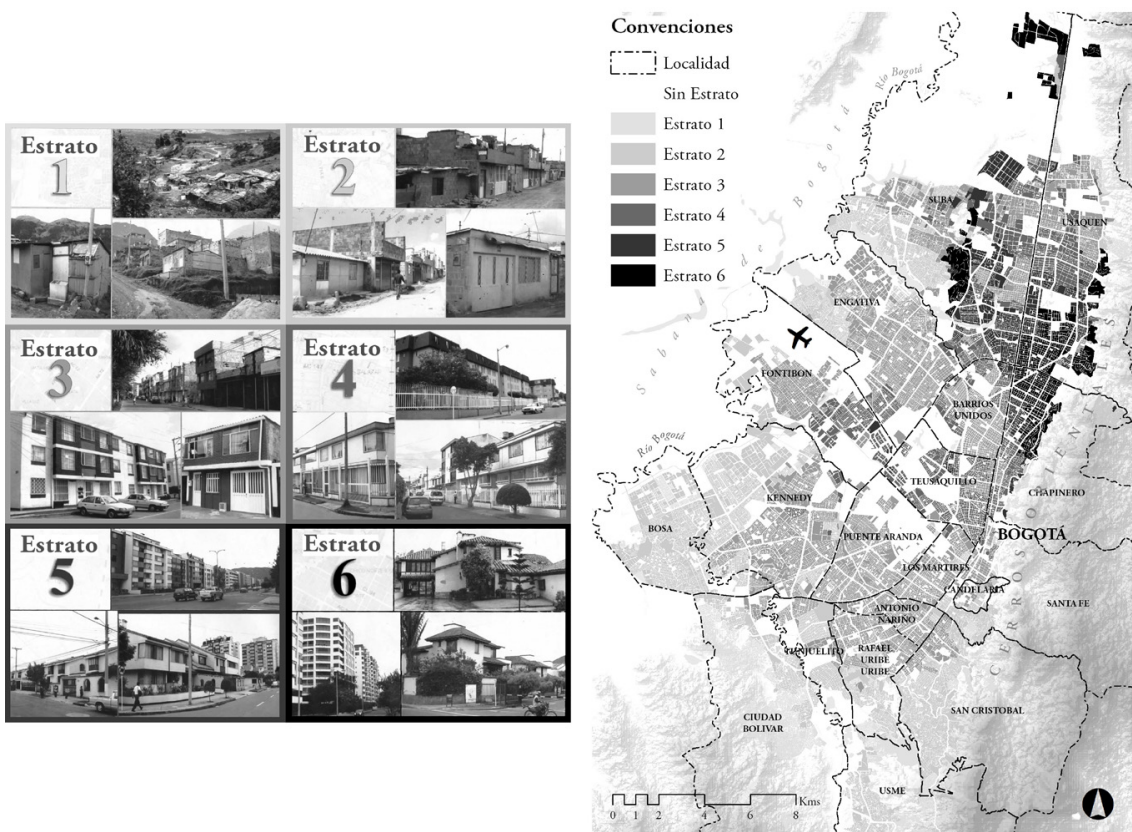

FUENTE DAPD (2004), LA ESTRATIFICACIÓN EN BOGOTÁ Y ESTUDIOS RELACIONADOS 1983-2004 (ANEXO); Y DECRETO 29I, DE 2013

Los autores coinciden en que esto se debe a deficiencias metodológicas, tales como la dificultad de cambiar de estrato un predio en una zona consolidada. La experiencia indica que el estrato solo cambia mayoritariamente para las nuevas construcciones en 
lotes previamente vacíos. Los cambios de estrato en áreas consolidadas se encuentran con una gran oposición por parte de las comunidades que las habitan, ya que en la práctica se les está retirando un subsidio. Contrariamente, los autores encontraron que la mayoría de las veces las comunidades piden que sus barrios sean reestratificados en un estrato menor, para disfrutar de mayores subsidios. El resultado de estos procesos es que la estratificación que se introdujo en 1999 en Bogotá ha cambiado muy poco más de quince años después. Como lo indicó una funcionaria de la Dirección de Estratificación de la SDP entrevistada para este estudio:

A los tres años se hizo la primera actualización y de ahí en adelante se han ido haciendo cada tres o cuatro años actualizaciones. Pero es para incorporar más que todo lo que va surgiendo, pues la ciudad va creciendo y van apareciendo nuevos barrios; entonces cada dos o tres años se incorpora el nuevo barrio y se revisan algunos pequeńos sectores. Pero, en general, la mayoría de la ciudad tiene hoy [2016] el mismo estrato que se le definió en el año 1996.

Por otro lado, la influencia de la estratificación socioeconómica en los patrones locales de segregación social ha sido estudiada a través de encuestas, entrevistas e historias de vida por parte de académicos en ciencias sociales. Específicamente, Uribe Mallarino (2008) encontró que los habitantes de Bogotá han construido percepciones sociales a través del indicador de estrato, el cual es recordado mes a mes a través de la factura de servicios públicos domiciliarios. Las investigaciones sociológicas indican que hay un fuerte sentido de apropiación del territorio, bien sea por parte de los habitantes de casas de autoconstrucción - frecuente en las áreas de estratos bajos - o dentro de conjuntos cerrados, característicos de las áreas de estratos medios y altos. Como resultado de esto, las personas se sienten desposeídas cuando se trasladan entre áreas de diferente estrato. Un ejemplo de esto se da cuando aquellas que habitaban en barrios de origen informal, al moverse a conjuntos cerrados, extrańan las áreas comerciales y de industria artesanal cercanas a la antigua vivienda. O bien está el caso de personas que habitaban conjuntos cerrados y se mueven a barrios de origen informal, y se sienten incómodas por el ruido y la congestión. Otras percepciones incluyen el sentimiento de que las áreas de estrato alto son más seguras, o que sus habitantes son menos amables que en las de bajo estrato. Como consecuencia de estos sentimientos de apropiación y desposesión, se percibió una baja movilidad entre estratos. Cuando se les preguntó a personas de distinto estrato acerca de lugares donde van a recrearse o divertirse, la mayoría manifestó visitar lugares "de su mismo estrato"; lo mismo ocurre en su preferencia para buscar pareja, lo que limita las instancias de encuentro entre personas de diferente nivel. Esto se ve acentuado, por ejemplo, por lo que ocurre entre las familias de los estrato 1 y 2 , que envían a sus hijos a escuelas públicas mayoritariamente, mientras los estratos 4, 5 y 6 los matriculan en escuelas privadas exclusivamente. Sin embargo, Uribe-Mallarino también encontró intercambios de beneficio mutuo, específicamente en las zonas de borde entre estratos altos y bajos, donde los pequeños comerciantes ubicados en estrato bajo se benefician de la afluencia de personas con mayor poder adquisitivo, y estas de la cercanía de posibles trabajadores. 


\section{La vigencia de la estratificación socioeconómica}

Las críticas expuestas en la sección anterior muestran que no hay acuerdo acerca de la evolución de esta política pública, la estratificación socioeconómica. Los estudios enfocados en el ámbito metodológico sugieren que la estratificación tiene vacíos en su metodología cuando se trata de determinar el nivel de ingreso de las familias; sin embargo, estudios sociológicos demuestran que ella es asimilada por la población como una representación de la clase social. Con el fin de ampliar la información acerca de tal conflicto, y para determinar si los estratos están correlacionados con una diferencia en el nivel educativo de la población, en este estudio se utilizaron los resultados de la última encuesta multipropósito aplicada en Bogotá el año 2014. El nivel de educación de la población es importante debido a que se ha demostrado que un nivel alto incrementa las diferencias sociales (Archer, Hutchings \& Ross, 2005), y ha sido utilizado por otros estudios en el ámbito latinoamericano para determinar los niveles de segregación socioespacial (Aguilar \& Mateos, 2011).

Los datos utilizados fueron los de la encuesta multipropósito 2014, debido a que el último censo en Colombia se realizó el año 2005 y, por ende, su información era muy antigua para compararla con las de las fuentes a las que se recurrió en este estudio. La encuesta multipropósito se realiza periódicamente por el Departamento Administrativo Nacional de Estadística (DANE) y la SDP, a un representativo $10 \%$ de la población de la ciudad a lo largo de los diferentes estratos y localidades. De la aplicada en 2014 se tomó la pregunta acerca del último nivel educativo aprobado para la población mayor a 25 años. Los resultados se muestran en la figura 2 a nivel de estrato y en su distribución espacial a lo largo de la ciudad. La gráfica de barras a la izquierda muestra que, en 2014 , el estrato se asociaba a diferencias en el nivel educativo de la población adulta. En los estratos 1 y 2, más del $80 \%$ de la población adulta alcanzó apenas una educación básica, primaria o secundaria. En el estrato 3, la población tiene diversos niveles educativos; en los estratos 4 y 5 la mayoría de población adulta (entre 60\% y 70\% del total) tiene nivel universitario; y el estrato 6 se destaca por tener una población donde casi el 50\% alcanza el nivel de posgrado.

A la derecha de la figura 2 se muestra un mapa con los porcentajes de población con nivel universitario o posgrado, que utiliza datos de la misma encuesta multipropósito 2014. En el mapa se evidencia cómo el área nororiental de la ciudad y el eje hacia el aeropuerto, donde se localizan las áreas de estratos 4, 5 y 6, tienen mayoritariamente población con nivel educativo superior. Las áreas perimetrales noroccidental y sur tienen menos del 50\% de penetración de la educación superior; y en la periferia occidental y sur, menos del $20 \%$ de la población indicó tener estudios superiores. 
FIGURA 2 | Estratificación y nivel educativo de la población mayor a 25 años (izq.) Porcentaje de población con nivel educativo universitario o posgrado (der.)
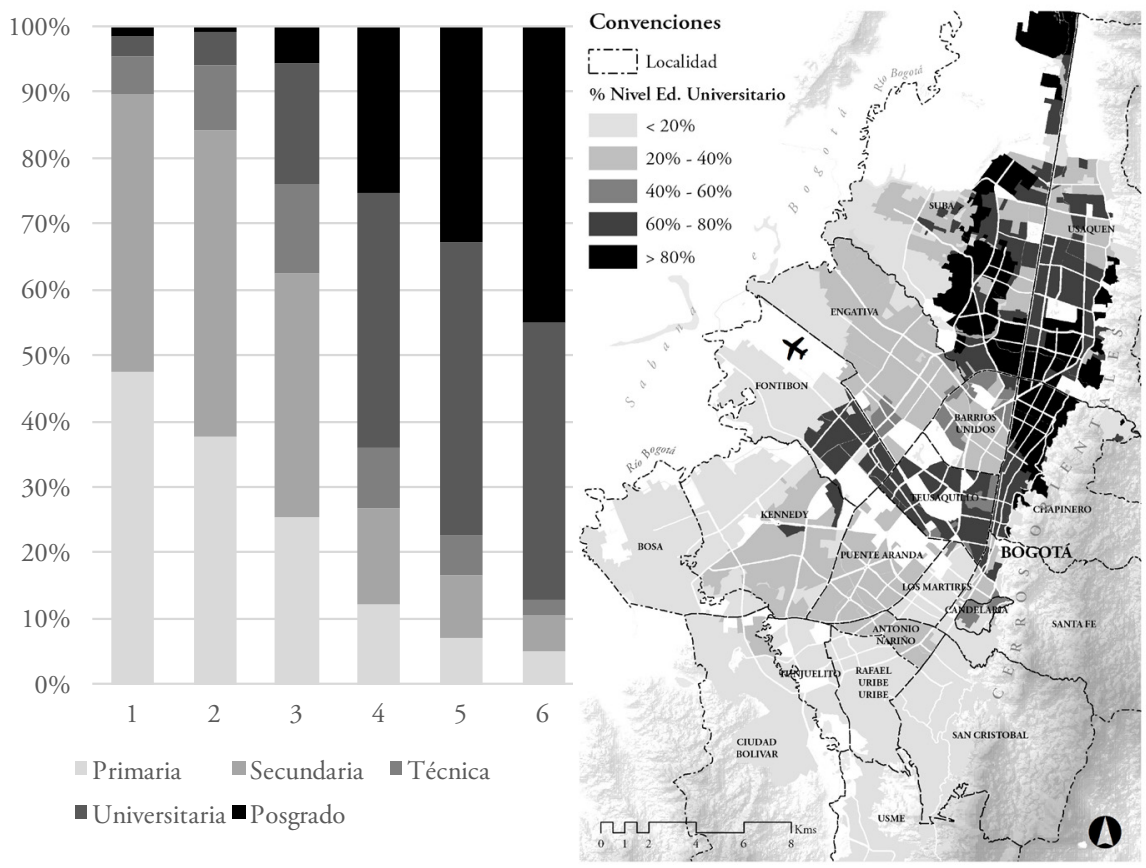

FUENTE ELABORACIÓN PROPIA CON BASE EN DANE (2O I 5, JULIO), ENCUESTA MULTIPROPÓSITO 20 I 4

\section{Estratificación socioeconómica y ordenamiento territorial}

La política de estratificación tiene una relación que puede resultar poco clara con la planeación según se lleva a cabo en el régimen colombiano de ordenamiento territorial. En la Ley 388 (Congreso de Colombia, 1997), que reglamenta el ordenamiento territorial en el país, los términos "estrato" o "estratificación" solo aparecen dos veces en artículos que reglamentan la prestación de los servicios públicos domiciliarios. El actual рот de Bogotá, compilado por el Decreto 190 de 2004, menciona que la estratificación es una de las variables necesarias de tener en cuenta para reglamentar los usos de suelo y edificabilidades en las áreas de planeación de escala local, llamadas Unidades de Planeación Zonal (UPZ), que son 117 a lo largo de la ciudad (Alcalde Mayor de Bogotá D.C., 2004, Art. 50). Sin embargo, pocas upZ contienen barrios de diferente estrato y su normativa solo tiene alcance local, por lo cual con este diseño normativo es imposible establecer una estrategia de estratificación socioeconómica y ordenamiento territorial a escala de ciudad. Más adelante se tratará este punto con mayor profundidad.

Como resultado de lo anterior, profesionales de la SDP encargados de trazar el nuevo рот de Bogotá manifestaron que percibían que la estratificación no era una variable de importancia para establecer la política de densificación de la ciudad, 
mientras otras variables, como el tamaño de los lotes, eran más importantes. Los profesionales manifestaron que la estratificación no afecta los patrones de uso del suelo o las alturas máximas permitidas; al respecto, señalaron que utilizan la estratificación más como una variable de diagnóstico que de planificación. Como indicó un consultor en planificación en una de las entrevistas realizadas: "El sector público ha visto siempre la estratificación como un resultado, pero no como instrumento de política. Como resultado de una manera de hacer operativo el manejo de esas áreas, pero no como un dato de base para tomar decisiones".

Sin embargo, la realidad que se vive en la ciudad parece ser otra. Por ejemplo, los patrones geográficos de densificación predio a predio de la ciudad parecen seguir el sistema de estratificación. La densificación predio a predio es una práctica común en Bogotá, debido al frecuente incremento de los límites de altura en la ciudad. Se ha convertido también en un método importante de desarrollo urbano, dada la escasez de suelo urbanizado en la ciudad, limitada por sistemas montañosos al oriente y sur y por el río Bogotá al occidente. Utilizando datos del censo nacional de 1993, Rincón Avellaneda (2004) identificó que en Bogotá la densificación predio a predio está relacionada con la estratificación. En las manzanas de mayor estrato (4, 5 y 6) hay una intensiva transformación morfológica, en que casas de dos o tres pisos pasan a edificios de cinco o seis; o cuando se hace integración predial, a edificios de diez o veinte pisos. Por el contrario, en las manzanas de estratos bajos $(1,2$ y 3$)$ hay una densificación informal progresiva, donde casas de autoconstrucción alcanzan tres, cuatro o cinco pisos a través de ampliaciones ilegales.

El discurso de la literatura local sobre avalúos de suelo y desarrollo inmobiliario también resalta la importancia de la estratificación para determinar las características y ubicación de productos de vivienda por parte de los promotores inmobiliarios. Como afirma Borrero Ochoa (2000), pionero de la enseńanza de avalúos prediales en Colombia: "El valor de la tierra va dependiendo del nivel social que la ocupe. La experiencia de nuestras ciudades indica que quien jalona el desarrollo es el estrato alto. A su alrededor tienden a agruparse los estratos medios. El sistema de planeación ubica a los niveles populares segregándolos de los estratos alto y medio" (p. 18)

Lo anterior apunta a una contradicción entre el discurso de la ley y de los profesionales en planificación, por una parte, y por otra lo que se vive a diario en el desarrollo inmobiliario de la ciudad, tal como lo identificó Rincón Avellaneda (2004). Esta contradicción se ve acentuada por la aparente intención de la reciente normativa urbana de eliminar la densificación predio a predio. El análisis urbanístico de la ciudad realizado en la década de 1990 como insumo del Decreto 190 determinó que la densificación predio a predio había causado un crecimiento desordenado de la ciudad, que no ha sido coherente con la oferta de infraestructura y servicios. Buena parte de estos efectos fueron atribuidos a la ausencia de la escala zonal de planeamiento (DAPD, 2000, p. 19). Como respuesta a esta problemática se implementaron los llamados Planes Parciales (pP). La norma del Decreto 190 incentivó que el crecimiento de la ciudad se ejecutara a través de los pp, herramientas de planificación que obligan a los promotores a realizar desarrollos completos de suficiente escala para proveer infraestructura de movilidad, servicios y espacio público coherente con las necesidades de la nueva población, y ello bien sea en la periferia 
(pp de modalidad Desarrollo), o dentro de la ciudad consolidada (pP de modalidad Renovación Urbana). Consecuente con esto, la norma del Decreto 190 establece varias áreas susceptibles de ser transformadas a través de pp, en el norte, el centro y el sur de la ciudad, y a lo largo de los diferentes estratos. Incluso en las áreas desarrolladas informalmente es posible la construcción en alturas de hasta ocho pisos en los ejes de movilidad, y la integración de predios para grandes proyectos de vivienda a través de otra herramienta, llamada Mejoramiento Integral, modalidad Intervención Reestructurante (Alcalde Mayor de Bogotá D.c., 2004, Art. 386).

Quince años después de implementados los pp, la experiencia indica que ello ha sido problemático. El sector privado ha desistido de muchos pp ante dificultades en su trámite administrativo, conflictos con las comunidades o escasez de suelo (Contreras-Ortiz, 2016; Mayorga-Lamouroux, 2012). Por ende, la ciudad sigue desarrollándose mayoritariamente predio a predio. Recientes estudios para la formulación de un nuevo рот indican que más del $60 \%$ de la construcción de la ciudad se ha continuado haciendo en esta modalidad a lo largo de los últimos años (Medellín \& Fernández, 2016; Ortiz, 2017). Específicamente, las normas que actualmente reglamentan la densificación predio a predio de la ciudad están consignadas a través de las UPZ. Esto enfatiza la actual importancia de analizar el patrón geográfico de la localización de las licencias de construcción de densificación predio a predio, y evaluar la normativa específica de cada unidad de planeación zonal.

\section{Resultados del análisis de localización de licencias de construcción 2010-2015}

Para la realización de este estudio, la SDP proporcionó una base de datos de todas las licencias de construcción otorgadas en la ciudad desde que se lleva un registro digital, el cual incluye la georreferenciación e información de uso solicitado, altura y área total por construir. Las licencias más antiguas correspondían a finales del año 2009 y las más recientes, al año en que se efectuó este estudio (2016). Con el fin de abarcar años completos, se seleccionaron todas las licencias dadas entre 2010 y 2015. Para identificar aquellas que contemplaban densificación predio a predio se utilizó como proxy todas las licencias que solicitaron alturas iguales o superiores a cuatro pisos. Igualmente se eliminaron del estudio las licencias de construcción en lotes con áreas menores a 100 o mayores a 5.000 metros cuadrados, y áreas construidas menores a 100 y mayores a 10.000 metros cuadrados. Esto con el fin de eliminar edificaciones singulares que pudieran contaminar la muestra y errores en la digitación de la información en la base de datos. Con este método se identificaron 2.060 licencias de construcción para usos del suelo residenciales y 236 licencias de construcción para usos no residenciales (oficina y comercio) a lo largo de la ciudad.

Posiblemente esta base de datos no incluye las numerosas ampliaciones verticales y horizontales realizadas informalmente a las viviendas por parte de los propietarios. Sin embargo, la información que contiene es importante, debido a que muestra los patrones de localización de proyectos del sector formal de producción de vivienda, el cual ha crecido y se ha consolidado en los últimos años. Igualmente, la ciudad ha mantenido esfuerzos importantes en el control a la construcción ilegal, y facilitado 
el acceso a permisos de construcción sin costo en barrios de origen informal, a través de la llamada "Curaduría Cero".

Mediante la georreferenciación de las licencias de construcción, se las ubicó en un plano de la ciudad. Se utilizó para ello un software de Sistemas de Información Geográfica y el mapa de referencia de datos espaciales de estratificación y manzanas de Bogotá obtenida en el sitio web del IDECA $^{1}$, correspondiente a diciembre de 2015. Este ejercicio permitió calcular la cantidad de área por construir en metros cuadrados solicitada en licencias de construcción para cada estrato entre 2010 y 2015, para usos de vivienda y de comercio y oficinas. Este resultado se comparó con el total de área y población en las manzanas de cada estrato, utilizando datos también suministrados por la SDP. Los resultados de este análisis se presentan en la figura 3 .

La gráfica de barras a la izquierda de la figura muestra — utilizando porcentajesel desbalance entre área, población y el otorgamiento de licencias de construcción para densificación predio a predio para vivienda y usos de oficinas y comercio entre 2010 y 2015 en Bogotá. Por ejemplo, como se puede apreciar en la gráfica, el estrato 6 , el más alto, que tiene apenas el $2 \%$ de la población de la ciudad y un $7 \%$ del total de área urbanizada, obtuvo el 30\% de toda el área de uso vivienda y un $41 \%$ del área en usos de oficinas y comercio. Igualmente, el estrato 5 , con un $2 \%$ de la población de la ciudad y un $6 \%$ del área urbana, obtuvo un $27 \%$ del área de vivienda y un $21 \%$ del área de oficinas y comercio. De manera opuesta, el estrato 2, con 41\% de la población y un 30\% del área urbana, obtuvo solo un $4 \%$ del área de uso vivienda y un $2 \%$ del área de uso oficinas y comercio. El estrato 1, el más bajo, con $9 \%$ de la población y un $10 \%$ del área urbana, no obtuvo un número significativo de área en los usos analizados que alcanzara al 1\% del total. Estos resultados muestran cómo la densificación predio a predio de la ciudad estuvo sesgada hacia los estratos altos 5 y 6. Estos constituyen 13\% del área total de la ciudad, pero recibieron en el periodo analizado un 57\% del total de área licenciada en vivienda y un $61 \%$ del total del área licenciada en oficinas y comercio.

El mapa en la figura 3, a la derecha, muestra la localización de las licencias de construcción de uso vivienda, clasificadas por la cantidad de área propuesta sobre un mapa de estratificación de la ciudad. En este mapa también se observa un patrón de desigualdad en la distribución de los proyectos de densificación. El mapa muestra un patrón de concentración de la mayoría de las licencias y de los más grandes proyectos en el nororiente de la ciudad, en áreas de estrato 4, 5 y 6 de las localidades de Chapinero y Usaquén. Se observan también algunos clústeres de proyectos en otras zonas al occidente y sur de la ciudad, pero estos poseen menor cantidad de proyectos y cada uno de ellos es de menor tamańo, principalmente en áreas de estrato 3 (medio), y en menor medida en estratos 1 y 2. 
FIGURA 3 | Densificación predio a predio y estrato, 2010-2015
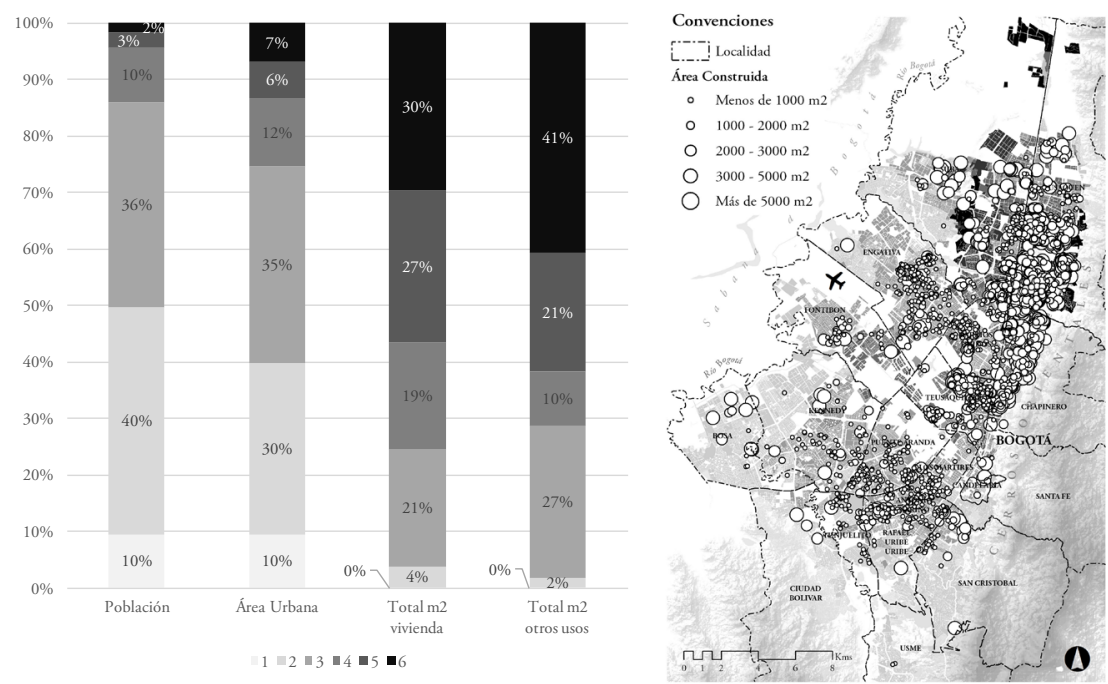

FUENTE ELABORACIÓN PROPIA CON BASE EN DATOS PROPORCIONADOS POR LA SDP

\section{La normativa local como facilitadora de una inversión sesgada}

El desbalance en la densificación predio a predio entre estratos y áreas de la ciudad sugirió la revisión de la normativa vigente de edificabilidad en el periodo analizado. Como se indicó anteriormente, el Decreto 190 establece que la normativa de uso de suelo, volumetría y alturas para la densificación predio a predio, debe ser establecida a través de las upz. Según la ley, estas herramientas deberían desarrollar cada una su normativa urbanística de acuerdo con un "Modelo de Ordenamiento Territorial" de mayor jerarquía, planteado en el Decreto 190.

El Decreto 190 estableció un Modelo de Ordenamiento Territorial, reproducido en la figura $4(\mathrm{~A})$, con tres piezas urbanas: 1) un centro metropolitano de alta densidad y uso múltiple que albergara las actividades terciarias y los equipamientos de mayor jerarquía; 2) un tejido residencial en el norte y en el sur estructurado a través de una serie de centralidades locales; y 3) unas áreas de expansión en el norte y en el sur. Algunos objetivos para consolidar este modelo incluyeron la limitación del crecimiento del centro metropolitano hacia el norte para reorientarlo hacia el eje occidental, donde se localiza el aeropuerto Eldorado, y así vincular mejor la ciudad con la región metropolitana. Paralelamente se determinó como objetivo recuperar la calidad urbanística del tejido residencial, restringiendo la localización de usos incompatibles con la vivienda (DAPD, 2000, p. 28).

Con el fin de trasladar el Modelo de Ordenamiento Territorial a la normativa local de las 117 upz, el plan delimitó una serie de "tratamientos". Los tratamientos urbanísticos se delimitan en zonas de ciudad con un determinado uso principal y nivel de consolidación, para facilitar la formulación de una normativa de uso del suelo y edificabilidad. Tales tratamientos tienen su origen en el pasado plan director 
de Bogotá, Acuerdo 7 de 1979 (DAPD, 1981), y actualmente está reglamentado su uso para todo el país (Congreso de Colombia, 1997). Según la ley colombiana, existen cinco tratamientos: (i) el tratamiento de "Conservación", diseñado para áreas consolidadas o de interés patrimonial, donde se mantiene la normativa inicial que dio origen a los barrios; (ii) el tratamiento de "Consolidación", para aplicación en áreas con potencial de densificación predio a predio y diversificación de usos del suelo; (iii) el tratamiento de "Mejoramiento Integral", diseńado para el mejoramiento de áreas con asentamientos de origen informal; (iv) el tratamiento de "Renovación Urbana", para incentivar el desarrollo en áreas en deterioro social y urbanístico; y (v) el tratamiento de "Desarrollo", para aplicar en las áreas que no han sido urbanizadas.

Desde la primera formulación de un РОт para Bogotá en 2000 hasta la fecha de este estudio, se han reglamentado 90 UpZ a través de procesos particulares de planificación y operaciones urbanas estratégicas. Las otras UPZ aún cuentan con la normativa del Acuerdo 6 de 1990, anterior al Decreto 190 de 2004. Para este estudio se analizó la totalidad de la normativa vigente, incluyendo los diferentes decretos de las 90 upZ reglamentadas y del Acuerdo 6, en los casos en que la UPZ no estuviera reglamentada, específicamente evaluando las alturas permitidas en las áreas con tratamiento de Consolidación. Los resultados se presentan en la figura 4 (B), donde se presenta las alturas máximas permitidas en los sectores con tratamiento de Consolidación, normativa que permite la densificación predio a predio. Las áreas en tono claro representan los lugares donde se permiten alturas máximas de tres pisos, al interior de los barrios, y con un tono un poco más oscuro aquellas donde se permite alturas mayores, en corredores locales de comercio. Estas áreas equivalen a las zonas de tejido residencial delineadas por el modelo de ordenamiento del Decreto 190. En tonos más oscuros se muestran los lugares de la ciudad donde se permiten alturas máximas entre seis y doce pisos. Estas áreas se corresponden con el centro metropolitano delineado por el Decreto 190. Las áreas en negro corresponden a las áreas donde la normativa de la UPZ permite una altura libre, solo limitada a través de índices de construcción ${ }^{2}$, que pueden llegar a cuatro o más.

En términos generales, el mapa muestra cómo la normativa de las UPZ estableció mayores límites de altura en el tejido residencial norte de la ciudad, específicamente en lugares clasificados en estratos 4, 5 y 6 . Llama especialmente la atención la zona oscura en la localidad de Usaquén de estrato 4 y 5 , donde la normativa de la UPZ estableció un área de alturas libres, en contravía de lo que indicaba el modelo de ordenamiento. Con base en esta figura se intuye que, además de la preferencia por la inversión en el nororiente de la ciudad por parte del sector privado, las normativas de edificabilidad de las UPZ -al menos parcialmente- diluyeron la estructura propuesta por el Decreto 190. Esto fue también confirmado a través de las entrevistas con profesionales locales en planificación:

Las normas que dieron las UPZ, al contrario, inhibieron el desarrollo adecuado de las centralidades como aglomeraciones de actividad económica, como se esperaba. Desdibujaron un poco también los sectores que propiamente queríamos que

2 El Índice de Construcción representa el número de veces que se puede multiplicar el área de un predio. La fórmula es la misma del índice Floor to Area Ratio (FAR) mencionado en la literatura de habla inglesa. 
siguieran ocupando, y más bien las dispersaron. Entonces hubo allí un desencuentro en lo que fue el proceso participativo de la norma con los grandes propósitos del plan.

Otros profesionales también mencionaron que estas contradicciones fueron facilitadas por la presión ejercida por los promotores inmobiliarios sobre los procesos locales de planificación de las upz. Por ejemplo, en la upz Chicó-Lago-Refugio en la localidad de Chapinero, que concentra estratos 5 y 6 , un profesional con experiencia en el proceso comentó: "Sí, probablemente hubo influencia (...). Ciento y pico de UPZ no las habíamos terminado de reglamentar y ya esa la habían reglamentado cuatro veces. ¡La arreglaron cuatro veces! Eso es una señal de presión, pero ¿̨de qué deriva la presión? Parte del mercado, los mercados presionan”.

FIGURA 4 | (a) Modelo de ordenamiento territorial; (b) Alturas permitidas tratamiento de consolidación
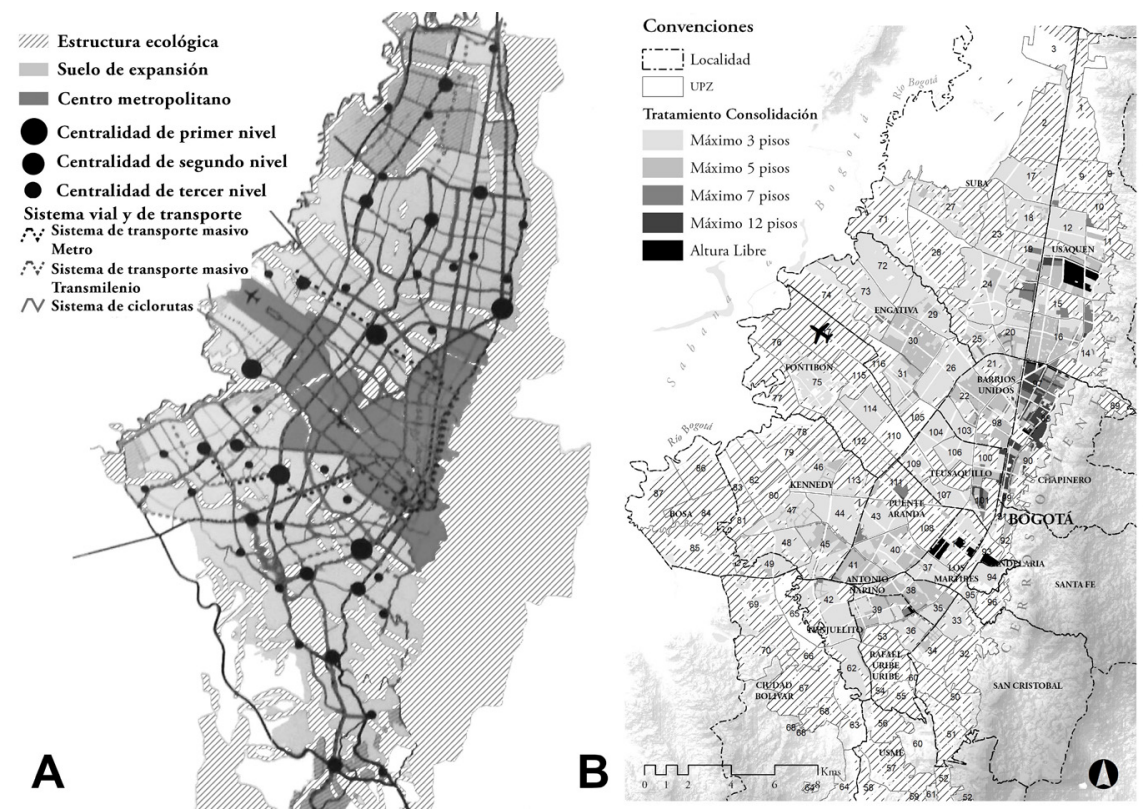

FUENTE (A) ELABORACIÓN PROPIA CON BASE EN DAPD (2000). (B) ELABORACIÓN PROPIA CON BASE EN DECRETO I 90 DE 2004 Y DECRETOS REGLAMENTARIOS DE LAS UPZ

Como resultado de lo anterior, entre los académicos locales en planificación existe consenso respecto de que la formulación de la normativa de uso de suelo y edificabilidad a través de las uPz fue un error. Esto, en buena medida, debido a la relación asimétrica entre el poder de persuasión de los promotores inmobiliarios y el de los profesionales que formularon la normativa de las UpZ. Como lo afirma otro profesional en planeación y académico local:

La embarramos con las upZ, se nos salieron un poco de las manos esas ciento y pico. Muchas de las UPZ no cumplen las ideas de planeación que se habían hecho en el plan original. [x] hizo como 20 upz. Yo le dije: "Yo creo en usted, no lo veo a usted 
cediendo ante un promotor para darle mayor edificabilidad a un predio", y eran todos esos predios en el norte. Me dijo: "Sí, pero había dificultades en ciertas cosas, regular las upZ es más difícil, pero con una ciudad de este tamaño, ¿̇usted cómo más lo hace? No hay mucho más que hacer".

El resultado principal de estos procesos, como muestran los mapas presentados en este estudio, fue que la densificación predio a predio de la ciudad no correspondió al modelo de ordenamiento establecido por el Decreto 190. Uno de los ejemplos de las nefastas consecuencias de estos procesos fue la densificación no planeada en la localidad de Usaquén, que muy pronto produjo la saturación de la infraestructura de movilidad y servicios públicos en la zona conocida como Cedritos. La normativa de esta uPZ que permitió alturas libres, reglamentada por el Decreto 271 (Alcalde Mayor de Bogotá D.C., 2005), produjo un auge de la actividad inmobiliaria en la zona donde existían conjuntos de casas unifamiliares construidas en las décadas de 1970 y 1980 . El sector se mostró al público en los medios como el área estratégica de desarrollo urbano de la ciudad, en contravía con lo establecido en el Decreto 190. Como lo demuestra este texto tomado de un artículo del periódico El Tiempo:

Se calcula que el 60 por ciento del sector está urbanizado con viviendas de estrato cuatro, lo que lo convierte en una buena opción para las parejas recién casadas y los universitarios o profesionales que están ingresando al mercado laboral. Es un sector tradicional y sobre todo un sitio muy residencial al que, desde hace 20 ańos le ha apostado la población profesional por factores que son recurrentes: la ubicación y el Estrato que les permite vivir a un nivel muy alto de manera más económica (Redacción El Tiempo, 2007).

Sin embargo, para 2012 el auge inmobiliario que había motivado el desarrollo de grandes proyectos de vivienda de diez, quince y hasta veinte pisos en la zona, empezó a tener un grave impacto en la infraestructura urbana del barrio, diseñada originalmente para casas suburbanas unifamiliares. Ese ańo, la Empresa de Acueducto y Alcantarillado de Bogotá pidió en una carta a la SDP no autorizar más licencias de construcción en la zona, ya que el sistema de alcantarillado estaba a punto de colapsar y podía producirse una crisis sanitaria (Redacción Bogotá, 2012). Esta comunicación pronto llevó a la organización de los vecinos en contra de nuevos proyectos inmobiliarios en el lugar. A poco andar no solo las redes de servicios públicos mostraban señales de saturación, sino que el aumento del tráfico en el barrio llevó al frecuente colapso de sus estrechas calles. Como lo expresó un académico local en un artículo titulado "Las vías de Cedritos llegaron al límite": "Cedritos es el sector de la ciudad que mejor refleja las consecuencias de lo que sucede cuando se incrementa la cantidad de residentes en un barrio sin que aumente la oferta de malla vial para soportar esta carga" (Cante, 2013).

Finalmente, para fines de 2012, a través de un nuevo decreto la sDP prohibió las edificaciones de más de seis pisos en la zona. Sin embargo, para esa fecha, más de cien nuevos proyectos de vivienda en altura habían sido licenciados en el barrio. Sus impactos tendrán que ser soportados por los antiguos y nuevos habitantes de la zona por muchos ańos, y los costos del mejoramiento de la infraestructura tendrán que ser asumidos por todos los bogotanos. 


\section{Conclusiones y discusión}

Este estudio muestra que en Bogotá existe un desarrollo urbano sesgado, al menos en parte, por la inversión privada dirigida a través del sistema de estratificación socioeconómica. Específicamente, sus resultados sugieren que la densificación de la ciudad en los últimos años no ha seguido el modelo establecido por el РОт, sino -al contrario- los patrones de segregación establecidos por la política de estratificación socioeconómica, basados en las diferencias de tipologías de vivienda y morfología urbana. El estudio también describe cómo esta distribución sesgada de la inversión es facilitada por la influencia de agentes del sector privado en el desarrollo de las normas urbanísticas.

De esta manera, los resultados muestran cómo el sector privado tiene una distribución sesgada de su inversión en la densificación de la ciudad de Bogotá. A pesar de que la identificación de este fenómeno no es una novedad, en el pasado no existía una información detallada para analizarla, como la que ofrece la base de datos geográfica de licencias de construcción compilada desde 2009. Más importante, este trabajo aporta evidencia acerca de cómo políticas públicas como la estratificación socioespacial y normas como las UPZ, formuladas desde el sector público, contribuyen a este desbalance. Consecuentemente, confirma las teorías de Blanco (2012) y Koch (2015) acerca del urbanismo en Colombia. Por un lado, la distribución de la inversión presentada demuestra que existe una segmentación del mercado no solo de vivienda, sino de usos comerciales y de servicios en la ciudad, evidencia de un discurso de "emprendimiento basado en la tierra", fundamentado en las profundas diferencias sociales en la ciudad. Igualmente, las normas urbanísticas diferenciadas de las UPZ, que desdibujan el modelo de ordenamiento del рот, son también síntomas de un "urbanismo arreglado" por el sector privado. Estos fenómenos han venido incidiendo en la profundización de la segregación social y el desajuste espacial en los últimos ańos, e -igual de grave- han ido disminuyendo la calidad de los servicios públicos e infraestructura en las áreas con mayor desarrollo.

Para la fecha de redacción de este artículo, la ciudad de Bogotá estaba en el proceso de formulación de un nuevo рот, que derogará las disposiciones del Decreto 190 y de las uPZ. Este proceso moderará la inversión privada en desarrollo urbano por lo menos por doce años y, por ende, deberá incluir una mejor visión sobre las diferentes desigualdades sociales en la planificación. Solo un profundo entendimiento de las diferencias de ingreso, nivel educativo, morfología urbana y tipologías de vivienda por parte de los profesionales en planeación podrá facilitar el desarrollo de normas urbanísticas que promuevan la equidad social y la integración de la ciudad. Igualmente, solo procesos de planeación más transparentes y con más participación ciudadana podrán controlar la influencia de intereses privados sobre la normativa urbana de la ciudad.

\section{Agradecimientos}

Este trabajo fue apoyado por la beca de investigación LUT I I I9 I del Instituto Lincoln de Políticas del Suelo. 


\section{Referencias bibliográficas}

Aguilar, A. G. \& Mateos, P. (2011). Diferenciación sociodemográfica del espacio urbano de la Ciudad de México. EURE, 37(110), 5-30. https://doi.org/10.4067/S025071612011000100001

Alcalde Mayor de Bogotá D.c. (2004). Decreto 190 de 2004, Plan de Ordenamiento Territorial de Bogotá D.C., Pub. L. No. 190. Por medio del cual se compilan las disposiciones contenidas en los Decretos Distritales 619 de 2000 y 469 de 2003 [Derogado por el art. 565, Decreto Distrital 364 de 2013]. En http://www.alcaldiabogota.gov.co/sisjur/ normas/Norma1.jsp?i=13935\#0

Alcalde Mayor de Bogotá D.c. (2005). Decreto 271 de 2005. Por el cual se reglamenta la Unidad de Planeamiento Zonal (Upz) No. 13, Los Cedros, ubicada en la Localidad de Usaquén. Fecha de entrada en vigencia: 11/08/2005. En http://www.alcaldiabogota. gov.co/sisjur/normas/Norma1.jsp?i=17340

Alcalde Mayor de Bogotá D.c. (2013). Decreto 291 de 2013. Por medio del cual se adoptan los resultados de la sexta actualización de la estratificación urbana de Bogotá D.C., para los inmuebles residenciales de la ciudad [Derogado por el Art. 8, Decreto Distrital 394 de 2017]. En http://www.alcaldiabogota.gov.co/sisjur/normas/Norma1.jsp?i=53589

Aliaga-Linares, L. \& Alvarez-Rivadulla, M. J. (2010). Segregación residencial en Bogotá a través del tiempo y diferentes escalas (Working Paper No. WP10LL1SP). Washington D.C.: Lincoln Institute of Land Policy. En https://bit.ly/2Guo1N5

Archer, L., Hutchings, M. \& Ross, A. [con C. Leathwood, R. Gilchrist \& D. Phillips]. (2005). Higher education and social class: Issues of exclusion and inclusion. Londres \& Nueva York: RoutledgeFalmer.

Blanco, A. G. (2012). Discourses of land allocation and natural property rights: Land entrepreneurialism and informal settlements in Bogotá, Colombia. Planning Theory, 11(1), 20-43. https://doi.org/10.1177/1473095211403124

Borrero-Ochoa, O. (2000). Avalúos de Inmuebles y Garantías. Bogotá: Bhandar.

Cante, K. (2013, septiembre 10). Las vías de Cedritos llegaron al límite. El Tiempo. En http:// www.eltiempo.com/archivo/documento/CMS-13054466

Congreso de Colombia. Ley 142 de 1994 (1994). Servicios Públicos Domiciliarios. Por la cual se establece el régimen de los servicios públicos domiciliarios y se dictan otras disposiciones. Fecha de entrada en vigencia: 11/07/1994. En http://www. alcaldiabogota.gov.co/sisjur/normas/Norma1.jsp?i=2752

Congreso de Colombia. Ley Orgánica de Ordenamiento Territorial, 388 (1997). Por la cual se modifica la Ley 9 de 1989, y la Ley 2 de 1991 y se dictan otras disposiciones. Fecha de expedición: 18/07/1997. En http://www.alcaldiabogota.gov.co/sisjur/normas/ Norma1.jsp?i=339

Contreras-Ortiz, Y. (2016). Sistema urbanístico en Bogotá: reglas, prácticas y resultados de los planes parciales de desarrollo 2000-2015. Cuadernos de Vivienda y Urbanismo, 9(17), 122-141. https://doi.org/10.11144/Javeriana.cvu9-17.subr

DAPD. (2000). Plan de Ordenamiento Territorial de Bogotá POT, Documento Resumen. Bogotá D.C.: DAPD.

Departamento Administrativo de Planeación Distrital (DAPD), Alcaldía Mayor, Bogotá. (1981). Ordenamiento y Administración del Espacio Urbano en Bogotá. Bogotá: DAPD. 
Departamento Administrativo de Planeación Distrital (DAPD), Alcaldía Mayor, Bogotá. (2000). Plan de Ordenamiento Territorial de Bogotá (pot), Documento Resumen. Bogotá: DAPD.

Departamento Administrativo de Planeación Distrital (DAPD), Alcaldía Mayor, Bogotá. (2004). La estratificación en Bogotá D.C. y estudios relacionados 1983-2004 (p. 145). Bogotá: Alcaldía Mayor de Bogotá D.c.

Departamento Administrativo Nacional de Estadística (DANE).(2015, julio). Encuesta multipropósito 2014. Bogotá: DANE. En https:/www.dane.gov.co/index.php/ estadisticas-por-tema/pobreza-y-condiciones-de-vida/encuesta-multiproposito. También en Encuesta multipropósito, Bogotá, Ciudad de estadísticas, Boletín 65 (abril 2015). En https://bit.ly/1P25FND

Redacción Bogotá. (2012, septiembre 23). Redes no dan abasto en Cedritos. El Tiempo. En http://www.eltiempo.com/archivo/documento/CMS-12247524

Griffin, E. \& Ford, L. (1980). A model of Latin American city structure. Geographical Review, 70(4), 397-422. https://doi.org/10.2307/214076

Guzmán, L. A., Oviedo, D. \& Bocarejo, J. P. (2017). City profile: The Bogotá Metropolitan Area that never was. Cities, 60, 202-215. https://doi.org/10.1016/j.cities.2016.09.004

Ibatá-Ceballos, Á. \& Torres-Arias, H. (2006). La estratificación urbana como indicador socioeconómico. Bitácora Urbano Territorial, 1(10), 214-221. En https://dialnet. unirioja.es/ejemplar/311649

Kain, J. F. (1968). Housing segregation, Negro employment, and metropolitan decentralization. The Quarterly Journal of Economics, 82(2), 175-197. https://doi.org/10.2307/1885893

Koch, F. (2015). The rules of the game and how to change them: Urban planning between formal and informal practices. A Colombian case study. International Planning Studies, 20(4), 407-423. https://doi.org/10.1080/13563475.2015.1068685

Mayorga-Lamouroux, J. (2012, septiembre 5). Limitaciones en la gestión de planes parciales en áreas de renovación urbana en la ciudad de Bogotá, D.C. Trabajo presentado para optar al Grado de Maestría en Planeación Urbana y Regional. Pontificia Universidad Javeriana, Facultad de Arquitectura y Diseño, Bogotá D.c. En http://repository.javeriana.edu.co/ handle/10554/2067

Medellín, P. \& Fernández, J. (2016). Tratamiento de Renovación Urbana, ¿un fracaso? En Universidad Nacional de Colombia, Instituto de Estudios Urbanos, Noticias. En http:// ieu.unal.edu.co/noticias-del-ieu/item/tratamiento-de-renovacion-urbana-un-fracaso

Ortiz, A. (2017, agosto). Planes de Ordenamiento Territorial. Caso de Estudio: POT Bogotá. Presentado en: Ordenamiento Territorial en Colombia: Alcances y Retos de los Planes de Ordenamiento Territorial, Cámara de Comercio Chapinero. Bogotá: Secretaría de Planeación. En https://bit.ly/2pDl2av

Presidente de la República de Colombia. Decreto 3069 de 1968 (1968). Departamento Administrativo para la Prosperidad Social. Decreto 3069 de 1968. Por el cual se crea la Junta Nacional de Tarifas de Servicios Públicos, se establecen los criterios básicos para la aprobación de los mismos y se dictan otras disposiciones. En https://bit.ly/2G2PHsI

Redacción El Tiempo. (2007, abril 28). Arquitectura para todos los gustos. El Tiempo. En http://www.eltiempo.com/archivo/documento/MAM-2470833

Rincón-Avellaneda, P. (2004). Análisis de los procesos de re-densificacion en Bogota ¿Una alternativa al crecimiento sostenible? Bitácora Urbano Territorial, 1(8), 82-92. En https://revistas.unal.edu.co/index.php/bitacora/article/view/18760 
Secretaría Distrital de Planeación (SDP), Bogotá. (2011a). Bogotá, Ciudad de Estadisticas, Boletín No. 42. Método Integrado de Pobreza 2011. Bogotá: sDP, Alcaldía Mayor de Bogotá. En goo.gl/Lw444n

Secretaría Distrital de Planeación (SDP), Bogotá. (2011b). Vivienda, Hogares y Personas por Estrato 2009, 2010 y 2011. Bogotá: sDp, Alcaldía Mayor de Bogotá. En https://goo. $\mathrm{gl} / 82 \mathrm{Hg} 8 \mathrm{v}$

Secretaría Distrital de Planeación (SDP), Bogotá. (2015). Balance de Gestión Vigencia 2015. Indicadores del Acuerdo 067/2002. Bogotá: sDP, Alcaldía Mayor de Bogotá.

Sepúlveda Rico, C. E., López Camacho, D. \& Gallego Acevedo, J. M. (eds.). (2014). Los limites de la estratificación, en busca de alternativas. Bogotá D.c.: Editorial Universidad del Rosario.

Suárez, M. \& Delgado, J. (2009). Is Mexico city polycentric? A trip attraction capacity approach. Urban Studies, 46(10), 2187-2211. https://doi.org/10.1177/0042098009339429

Suárez-Lastra, M. \& Delgado-Campos, J. (2007). Estructura y eficiencia urbanas. Accesibilidad a empleos, localización residencial e ingreso en la ZMCM 1990-2000. Economía, Sociedady Territorio, 6(23), 693-724. En https://est.cmq.edu.mx/index.php/est/article/view/254/260

Uribe-Mallarino, C. (2008). Estratificación social en Bogotá: de la política pública a la dinámica de la segregación social. Universitas Humanistica, 65(65), 139-171. En http://revistas. javeriana.edu.co/index.php/univhumanistica/article/view/2245 